\title{
Research on Enhancing the Effectiveness of Staff-Training in Private Enterprise
}

\author{
Yang $\mathrm{Hu}^{1,2}$, Xinrong Yang ${ }^{1}$ \\ ${ }^{1}$ School of Economics and Management, Hunan Institute of Science and Technology, Yueyang, China; ${ }^{2}$ School of Economics and \\ Management, Wuhan University, Wuhan, China. \\ Email: drhuyang@163.com
}

Received September $25^{\text {th }}, 2009$; revised October $29^{\text {th }}, 2009$; accepted November $31^{\text {st }}, 2009$.

\begin{abstract}
In 21 st century, the enterprise competition is a comprehensive competition based on talent, capital, technology and energy. Especially, among all these elements, talent is the most important one. How to culture and set up the enterprise's own personnel training system is the foundation to improve the business competition strength. Training is one of the best approaches, while how to apply the training contents in practical work and to achieve the expected goal, has great economic and social significance to help Private Enterprise develop much better. According to the relevant theories of staff training, this paper indicated that it was meaningful to enhance the effectiveness of staff training in Private Enterprise after analyzing its current situation of staff training. Meanwhile, it also summed up the practical training procedures, particularly for the Private Enterprise, and provided some constructive suggestions to establish and to perfect the efficient training system in China.
\end{abstract}

Keywords: Private Enterprise, Staff training, Effectiveness

\section{The Significance of Enhancing the Effectiveness of Staff Training in Private Enterprise}

In view of the present situation, it is one of sensible choices to improve the comprehensive strength of private business via enhancing the training effectiveness. Whether considering the recent financial crisis or concerning about the business long-term development, enhancing the training effectiveness will become the booster for business development.

\subsection{To Help Revitalize the National Economy and Boost Chinese Confidence}

At present, the private economy has surpassed more than $50 \%$ of the total GDP of the National economy, solved more than $85 \%$ of employment problems, and settled more than $50 \%$ of the financial revenue. It has become a regional economy, especially as an indispensable support of County economy, however, the average life expectancy of China Private Enterprise is only 3.5 years. Faced with such an urgent reality, government is increasingly attaching importance to support the development of private enterprise. Beside policies, capital and other external supports, it is more important to help private enterprise to establish scientific management system and educating mechanism.

Private enterprise must realize that enhancing the effectiveness of staff training is not only the natural choice for enterprise itself development, but also the requirement of the times to revitalize the National economy and fulfill social responsibility. In view of the microenvironment, the improvement of enterprise-wide learning ability, will greatly enhance the enterprise competitiveness and stimulate innovation capability. To build a loyal, professional, and efficient management team and implementation team, so that the private business will go further in the future development.

\subsection{To be Favorable for Getting Rid of Financial Crisis and Realizing Leapfrog Development}

The global financial tsunami induced by the American sub-prime crisis, presented great challenges to the advanced private business as never before. Since China has a vast domestic market, the Chinese economy has maintained stable under the government's strong macro-control. People are full of confidence that Chinese economy will be the first to recover in the near future. The private business as an important component of Chinese economy, it should seize the opportunity to accumulate strength through various forms of learning, to expand and enhance the overall level of business, so as to adapt to a 
more intense, more standardized, and more sustainable development competition.

\subsection{To Accord with the Private Business's Own Development Requirements}

Most Private enterprises pay little attention to staff training of knowledge and skills, which is a common problem nowadays in private enterprises. As many enterprises were just in the form of workshop at their prior periods, mostly depending on emotional management, and practitioners were of low quality and poor skills. As corporate manager always wanted to reduce costs, very little money was invested in staff training, so that once the business turned to be bigger and stronger, some employees would be uncomfortable and work under pressure, especially the sales department, without proper training, it would be completely impossible to have staff sales achievement in proportion to his selling skills. In the limited time, use of the limited resources, greater enhancement of the training effectiveness, will greatly promote the development of private business.

\section{The Chinese Private Business Status and Problems of Staff Training}

At present, the employee training situation in Chinese private business is not optimistic. It can not imitate the practice in the major companies, which can run their own training schools or virtual educating. It is also very restricted to get training experiences from other medium and small enterprises.

\subsection{Staff Training is Lack of Scientific Planning and Execution is Poor}

Now the effect of staff training in Chinese private business does not perform well, since the lack of social services in the appropriate training market, or because these companies have not groped an effective approach. As staff training can not keep up, private enterprises always can only focus on "hardware" investments, but not improving the staff quality at the same time, resulting in enterprise "software" development can not keep up with "hardware" development demands. As hardware and software do not combine well, the effective result of investment in technological upgrading is not satisfactory. As a result, private enterprises are increasingly feeling complicated with staff training, realize that they can not neglect it, but have no idea how to start with it, so that there is an acute shortage of staff training activities, even form a vicious circle in human resources management.

Most private enterprises lack of scientific norms in the annual training plans. Mostly, personnel departments and trainees just go through the motions to complete task. Especially, in the production and sale type-based enterprises, all the members work hard to make achievement in the busy-season. Everything is achievement-oriented, so that no more time to talk about training. Only in the off-season, it will do training symbolically as a way of regulating work, even if doing annual training plan, the final curve will be also performed only in the form of a few random lectures, which is a fundamental departure from the purpose of training.

\subsection{Private Business Employees and Managers are of Low Quality}

The employees in private business are of lower literacy, lower level of education, and lower quality than the ones in state-owned enterprises and foreign enterprises. Based on an investment in private business in certain place, found that education situation in private employees are really worrisome: primary $17.76 \%$, middle school $45.74 \%$, high school $32.24 \%$, tertiary and higher $4.26 \%$; while the distribution of age structure, the young people (30 years of age) are in the vast majority of private enterprises employees, reaching $62.92 \%$. 30-40 years of age are accounted for $21.07 \%$, two together are accounted for $83.99 \%$. Private enterprises in other places also generally like this case, but in the technology-inten- sive private enterprises, the situation will be better, but young people in the workforce and the proportion are the same.

In addition, a considerable number of private enterprises do not have human resource management departments, the ones possess of human resources management department, of which the managers' quality and ability are not high. Private enterprises do not have high-quality human resource management professionals, and human resources managers of low quality fail to reflect the role of human resource management, so even simply ignore the human resource management, it would go so far as to have not a full-time Personnel Administrator in an enterprise of thousands of people. While in Europe enterprises with the same scale, most of them employ more than 10 human resource practitioners.

\subsection{A Serious Shortage of Investment in Staff Training}

At present, the Chinese private business has already exceeded $60 \%$ of the total number of Chinese companies, which is the largest business group in China, accounting for more than $50 \%$ share of GDP. However, enterprise investment in staff training is relatively low: only $8 \%$ of the companies, of which the enterprise investment is accounted for above 3\%o-5\%o of the total sales incomes, while $48.2 \%$ of enterprises, of which the enterprise investment is accounted for less than $0.5 \%$ of total sales incomes. In the view of enterprise development and the actual needs for talent competition, the low-input in training will seriously affect their quality.

As many Private businesses suspect employees loyalty, them only focus on the use of employees, without attention to training, are only willing to spend money on talent, 
not willing to invest in training personnel. As a staff, he does not only consider the issue today to earn a living, but also consider the future earning capacity, if he felt working conditions not conducive to a better future development, he would be reluctant to make long-term stay, and to leave once there is a suitable opportunity. At this it will form a vicious circle of human resources management.

\subsection{Staff Training Form in Chinese Private Business is Raw and Single}

Private businesses basically use the traditional training methods such as seminars, conferences and other forms, which are really boring. By a survey of staff training in private enterprises, found that private business staff training was still quite raw. Front-line operator training was mainly aimed at new staff, only $38 \%$ of front-line operational employees had received pre-job training, and it was mainly through meetings and the form of one by one, of which the main contents were business history, post basic skills, safety awareness and quality consciousness. The training time was 1-2 days. Most front-line employees were just simply ordered by the master, and then directed by old workers, to become familiar with jobs within a week or so. Some employees even immediately got appointment without any training. Managers complained that staff quality was too low, someone tried to train the front-line staff in way of courses, but staffs couldn't understand.

The managers above middle level, whether private owner members or non-private owner members, hardly get any training, despite the private business owners are usually discontent their managers. Private enterprises are accustomed to ready-made talent recruitment, only a handful of managers who are wished to be reused or to be promoted have training opportunities. The full-time learning opportunities are naturally very small, usually only when companies will introduce new equipment or new technology, and such opportunities are always given to private owner members. As government's request, the financial members usually have to participate in training organized by the relevant government departments, training and study time is about two weeks.

\subsection{The Lack of Scientific Evaluation System and a Low Conversion Rate}

Private business almost never make training assessment, private business owners may think that training can bring little or no return on investment; managers and trainers may lack of the expertise to conduct training assessment; managers and trainers do not want to assess the input time and energy; private business itself is lack of staff training and does not regulate, nor taken seriously. It is precisely because of no training assessment; the efficiency of conversion from training contents into practical productive is very low, obviously, the training will not be able to play its expected role. Managers believe that the training led to little practical significance, the staffs think that enterprises do not attach importance to staff development. In a long time, enterprise management will result in errors, reducing the staff-to-business sense of belonging, or even quit.

\section{The Countermeasure to Enhance the Effectiveness of Staff Training in Private Business}

After the completion of start-up, most of the private businesses enter the most dangerous growth stage. One of the roots is there is not a clear development strategy in enterprise, when the business scale is keeping expanding, the management can not control it. Enterprises shortlived and disappear instantly. As private business managers, it is required to firstly clear business development strategy and objectives, then gradually introduce and implement the scientific systemic management systems and tools. The training and management are mutually reinforcing, both cooperation and coordination, will accelerate the steady growth in business.

\subsection{To Establish the Correct Concept of Staff Training, Increase Investment in Human Capital}

In connected with the private business problems in personnel quality and staff training, firstly, it should focus on the personnel training and reserves. Through the methods of the training and introduction of talent, etc, it can make up the disadvantage of a relatively low overall staff quality. As management team, it is more required to enhance learning, to master the systematic and practical management knowledge, skills, and enhance the pattern of operation and management as soon as possible. If conditions permit, it should be appropriate to use professional managers in the enterprise operation and management. In addition, the reasonable investment to ensure the smooth conduct of training, private enterprises must be willing to invest in training, to improve the training budget. Considering the current status of private business, learn from the most advanced training concepts to effectively enhance the effectiveness of staff training in private business.

\subsection{To Develop Practical Training Programs and Goals}

There should be plans and objectives in training, it must proceed from the corporate strategy, and corporate culture characterized by a combination, so as to meet both of organization and staff needs.

Training program development should be modeled according to enterprise development plans, be fit to it so as to become business forward boosters. For private business, with long-term development goals, there must 
be an assorted training program to promote the development of enterprises. In addition, the process of planning is also the process of excavation needs. Refine the needs of enterprises and employees in order to enable the training targets.

At the same time, before the start of training, it should set its goal to achieve according to the development strategies. Not only convenient for the effectiveness of training to do tracking assessment, but also can enhance the trainees self-confidence, promote training carried out smoothly.

\subsection{To Combine the Training with Staff Career Planning}

As private business, it should focus on human resource development and management, preparing for enterprise long-term development to accumulate enterprise personnel. The training is important content in human resources development and management. Companies must combine staff career planning into the training plans, promote staff active learning, and apply training contents to the actual work, only need to well lead the strategy and direction. Set up training programs in turn, will get the psychological identity from staffs, stimulate them selfinterest in learning, and promote training to achieve better results. If the staff performance is improved, the entire team performance will be followed to be improved, so as to achieve the enterprise's growth. A good learning atmosphere will be good to promote training to carry out the work; otherwise, will be constrained to carry out the training and enterprise development and growth. Private enterprises must not only to strengthen the hardware, but also should pay attention to soft power enhancement.

\subsection{To Design the Suitable Training Contents}

After set up good training programs and goals, it's required to design the suitable training contents according to the actual needs of private enterprise, also including training methods and choosing trainers.

1) Select appropriate training methods

For training, the popular is not the best, but only suitable for enterprises actual situation is the most important criterion. First, for the training methods, companies can have a variety of options, mainly divided into two categories, namely house training and external training. Can either choose the traditional classroom teaching model, or can use video to expand indoor training, outdoor training, such a variety of ways to expand.

2) Select appropriate courses

For course selection, training directors should not be affected by the trend, but have to keep calm and rational choice. It is necessary to take into account the role of the curriculum of business, but also take into account the cost of the training budgets. Ultimately must be clear that effective curriculum should not only actual combat, but also minimizing the costs.

3) Select appropriate trainers

With a good curriculum, but also have to select a good trainer, it must be cautious no matter from internal or external. Companies do not have to choose the trainer of big name, but to learn more about the trainer's knowledge, experience, and personality charms. And then compare it with the enterprise training culture whether fit or not, this will make training more effective.

4) Prepare the necessary tools for training, equipment and venues.

In order to successfully carry out the training to reach the desired objectives, training requirements should be well prepared. Used properly, can not only stimulate the enthusiasm of staff, but also help to strengthen the effectiveness of training.

\subsection{To Emphasize on Training Conversion}

The most crucial aspect of training is how to apply the content of the training and to promote the work of the actual performance improvement. The errors of this part will fall short of the whole training, but also a serious impact on the follow-up implementation of the training program. As enterprise, it is required to fulfill the following aspects:

1) Make good unity of the training spirit in the ideological aspect

Trainees must be noted with learning objectives before training, training organizing department or full trainees should take sharing experience and seminars. Training department should give trainees the appropriate learning tasks, to enable participants possess of the learning purpose. After training, trainees are the light of their own learning experience and corporate practice, work-sharing discussions with the staff, firstly make good unity of the training spirit in the ideological aspect.

2) Learn to meet practical needs in the applications aspect

It should provide the opportunities of applying learned skills in various forms for the trainees. Training contents are based on the corporate strategic planning to develop, and enterprises have to provide more application opportunities as possible. Managers have to track the implementation, such as by methods of trainees' feedback. And it is to be combined with the staff performance appraisal and work responsibilities together, to develop a formal implementation of the provisions, promote enterprises to form the learning atmosphere of to meet practical needs.

\subsection{Do Well the Effectiveness Assessment and Feedback}

For the training effectiveness assessment, there are many methods and practical tools. As to the status of Chinese private business and the effect in the actual training assessment, integrate a set of suitable methodology for training assessment in private business: 
1) Identify the assessing level

Enterprises assessment level is of 1-4, including reflecting level, learning level, behavioral level, and results level. First-level mainly uses the questionnaire, interviews of participant, observation and other methods; second level mainly include examinations in courses; third level, investigate trainees three months after training, according to workload and changes in the performance; fourth level, will be linked to performance appraisal, if trainees can achieve the standards of post-work requirements, then the training is effective.

2) Select the assessment methods

Training assessment methods, including testing methods, behavioral observation, target achievement of assessment method, cost-benefit assessment methods, learning reporting of the assessment method, assessment method. Private enterprises should make flexible choice in conjunction with training practice.
3) Emphasis on the application of assessment data and feedback.

With the improvement of enterprise training mechanisms, advocacy private business sum up in line with job responsibilities of the competent model, construct a good foundation for the smooth operation and management work, and for the standardized and scientific management system. Completion of the training does not mean the end of training. As a manager, should considerate from business global development, based on the training evaluation data, sum up each advantage and disadvantage of training to provide the most useful supplement for next time. To make the training work can be carried out more smoothly, gradually form a complete and efficient training system and promote management system to be more structure and maturity.

From the above, sum up the operation flow as Figure 1 for practical training system in private enterprise:

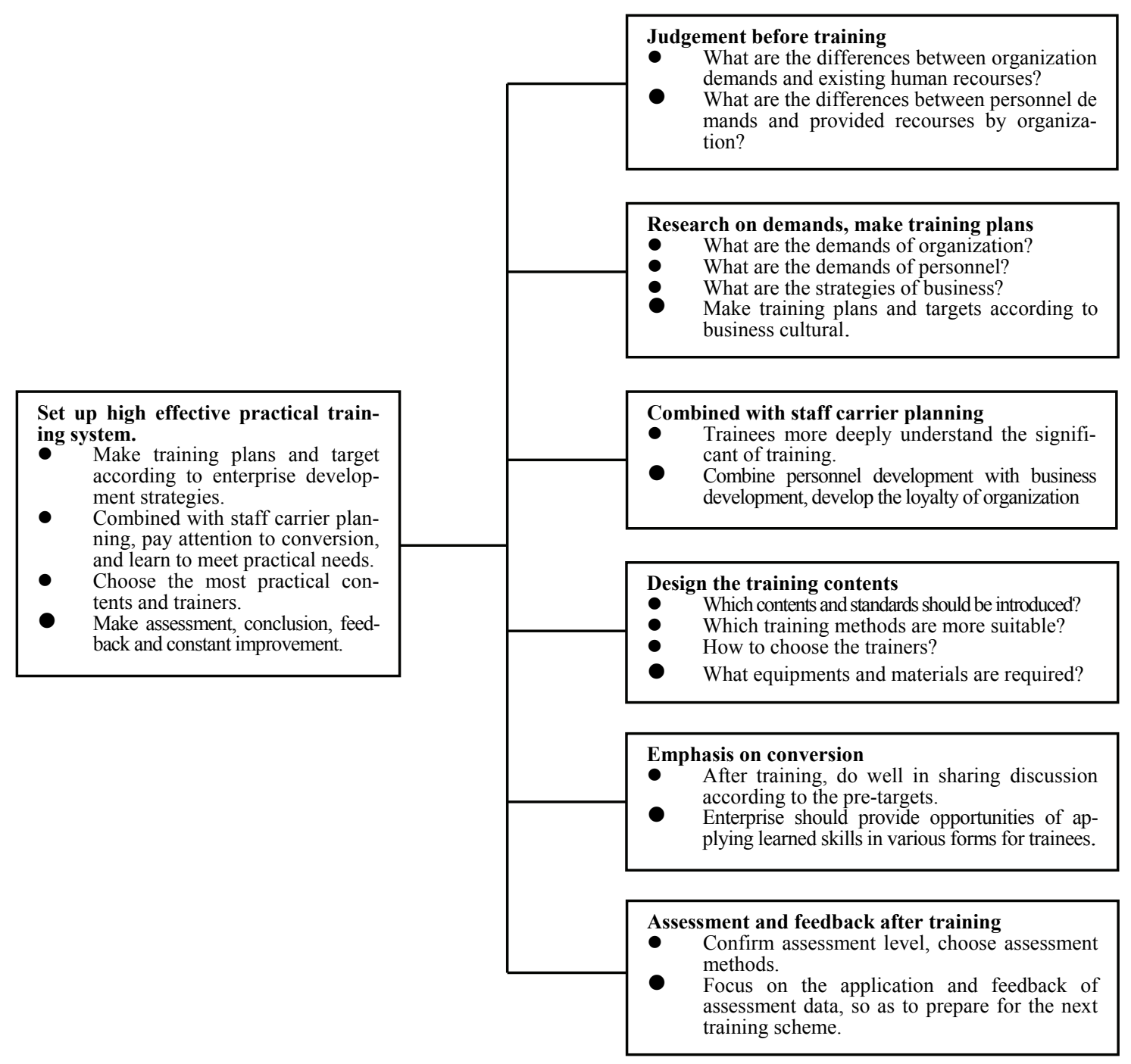

Figure 1. The operation flow of practical training system 


\section{Conclusions}

In the view of the enterprise organization forms, more and more private enterprises have adopted the modern enterprise system. From the development model perspective, the private enterprises have been gradually expanding from pure pursuit of quantity to the operational scale, technology level, product quantity and quality, high effective management, cost-effective direction. Training has been more and more concerned by private enterprises, in-depth research on enhancing the effectiveness of training in private business, is bound to provide a good reference and protection for the rapid development of private enterprises. To change the short life expectancy of private business, so that it can avoid market risks, develop to standardization, persist, and large-scale direction.

\section{Acknowledgements}

This paper is supported by China Postdoctoral Science Foundation (supporting ID: 20090460995).

\section{REFERENCES}

[1] F. Y. Liu, "Complete concept of human resources to human capital, the concept of change," China Human Resources Development, No. 10, 2007.

[2] D. K. Han and Y. He, et al, "Strengthening the manual cost accounting and control measures," Accounting research, No. 1, 2007.
[3] "Chinese enterprise's competitiveness report-Profitability and Competitiveness," Chinese Academy of Social Sciences Institute of Industrial Economics write: 2007 Enterprise Blue Book, 2007.

[4] Y. Hu and G. H. Liu, "The analysis and management measures of staff turnover under TBC," China Human Resources Development, No. 1, 2007.

[5] T. Y. Raymond, P. Keri, and K. George, "Zero timeinnovation strategy of an immediate response to customer demands," Electronic Industry Press, 2002 edition.

[6] Y. Hu and B. Hu, "Research of real-time incentive mechanism zero-time enterprise-oriented," Research Management, No. 4, 2007.

[7] M. Henry and Y. Q. Chen, "Strategic skill," IT managers in the world, No. 4, 2004.

[8] R. Q. Chen, "Research of new technologies and new methods in operation management time-based competition," An Application Report of National Natural Science Foundation, September 2003.

[9] B. S. Zhou and D. P. Fan, "Zero Time: The concept of enterprise in 21st century," China Mechanical Engineering, No. 2, 2000.

[10] N. J. Allen and J. P. Meyer, "The measure meat and antecedents of affective, continuance and normative commitment to the organization," Journal of Occupational Psychology, Vol. 63, pp. 1-18, 1990. 